\title{
An Indoor Ultrasonic Positioning System with Fast TOA Approach in Distance Space
}

\author{
Dawei Cai \\ Iwate Prefectural University \\ Iwate, Japan 020-0193
}

\begin{abstract}
With the rapid increase in guide services by smartphone or mobile terminal, demand for positioning has increased especially in complex indoor environment which often needs to determine the location information of the mobile terminal. In order to provide indoor positioning for real-time guide service that is on a mobile terminal platform, a new indoor positioning approach is proposed in this paper. We introduced a new concept of distance space and proposed a hybrid computation architecture which is divided into offline computation and online computation, to obtain a good realtime performance with less computation time. Here, an offline processing that may be complex computation will produce a numerical DB for a specified spot area, and an online computation with very simple computation is only for detecting the spot area. The offline computation is carried out in a high performance personal computer environment, and the DB data result is sent to NAND memory in a mobile terminal for online use. The online computation that will not cost much computation resource is run in an embedded platform that may have less computation ability. The simulation experimental results demonstrates that the detecting error is only within $5 \mathrm{~cm}$ and the computation speed was improved greatly.
\end{abstract}

\section{General Terms}

System Development

\section{Keywords}

Indoor Positioning, TOA, Space Mapping, Indoor Ultrasonic Positioning System, Smart Tourist Guide

\section{INTRODUCTION}

With the continuous development of wireless communication technologies and intelligent mobile terminals, location services have been rapidly popularized, and wireless positioning technologies have been widely used in transportation, logistics, sightseeing, and people's livelihood. In an outdoor environment, the Global Position System (GPS) locates the user nodes through satellite timing and ranging, and has the advantages of high positioning accuracy, good real-time performance, and strong antiinterference ability. Compared with the outdoor environment, the indoor environment is more complicated, the signal transmission is easily blocked by people, walls and other obstacles, the positioning difficulty is increased, the GPS capture satellite signal is rather weak, and its positioning performance cannot meet the actual people's production activity positioning accuracy requirements. Therefore, how to provide high-precision indoor positioning method has become a hot spot in the research of wireless positioning technology.

Aiming at the problem of wireless indoor positioning, many scholars and researchers have conducted much research and proposed many effective indoor positioning algorithms. According to the positioning principle, indoor positioning algorithms are mainly divided into two categories: time ranging positioning and Received Signal Strength Indication (RSSI) positioning. Timebased ranging methods mainly include: Time of Arrival (TOA) positioning, Time Difference of Arrival (TDOA) positioning, etc. The positioning algorithm based on TOA has defects such as noise sensitivity, multipath reflection and inaccurate of reference clock, large error of distance estimation. The positioning algorithm based on TDOA requires synchronization between devices. However, due to the limited indoor distance, the measured error of distance is large. They require the support of other auxiliary devices during the positioning process, so these algorithms are difficult to achieve effectively for indoor positioning application.

The positioning approach based on RSSI is as follows: firstly, the receiving node obtains the signal strength from the transmitting node, and calculates the signal propagation loss based on the strength of the signal received, and gets the distance between the two nodes based on a transmission loss model. Finally, the position of the node can be calculated by appropriate algorithm or approach. Compared with the time test distance positioning algorithm, it does not need to add additional hardware equipment, which is simple and easy to implement, and has low cost. It can expand the application range of the positioning system to the building group and the indoor, thus becoming the main research direction of indoor positioning. However, in the actual indoor environment, the signal propagation process is affected by environmental factors such as propagation direction, communication multipath phenomenon, and indoor structure. The RSSI value of the AP is not fixed and has time-varying. If the RSS value of the AP is directly used as input, the indoor environment is realized. Positioning results in large indoor positioning error and low precision.

However, electromagnetic waves propagate at the speed of light, so high accurate time measurement is required, and the positioning error is as large as several meters. For this reason, it is unsuitable for highly accurate positioning in indoor spaces. Positioning system 
using ultrasonic waves is limited to near-distance positioning application within several tens of meters, but since the sound speed is about $340 \mathrm{~m} / \mathrm{s}$, higher accurate positioning is possible. In the exhibition guide application for museums and art galleries, To determine the vicinity of the exhibit area, it is normally required that the positioning error should be within several tens of centimeters. @In this research, in order to realize low-cost, low power consumption and high-speed real-time positioning, a positioning method using high frequency sounds or ultrasonic waves is adopted.

With both sound signals and radio waves, we can get distance information between transmitting node and receiving node. In order to calculate the position estimation by the three point positioning principle, complicated calculation to solve the nonlinear simultaneous equation is necessary. In a mobile terminal driven by a battery whose capacity is limited, in order to complete such a complicated calculation, it is necessary to shorten the terminal utilization time with a long calculation time and high power consumption, or to achieve sufficient real-time performance. It is very important to develop high precision and real time characteristics for mobile information terminals and low cost indoor location estimation technology. Aiming at realization of automatic guidance service for tourists using high precision indoor position information of several centimeters, we focused on the mapping relation of the distance space composed of the position of the object and the distance from each sound source, Research and development on low-complexity indoor position estimation method will be carried out.

In this research, we introduce the concept of distance space and propose a unique approach to avoid complicated calculation to solve this nonlinear simultaneous equation. In this approach, a complicated and computationally intensive process is performed beforehand, and the coordinates of the location estimation area are recorded in a computer memory in a matrix form.

When estimating the position at the site, calculate the data recorded in the memory with its own search algorithm, and estimate the position with high speed and high accuracy. Unlike the viewpoint of the conventional method, the proposed method divides the calculation processing of position estimation into offline precalculation and on-line calculation, and greatly reduces online calculation amount. Therefore, the speed of position estimation calculation is achieved. In the process of position estimation, there is an advantage in that position estimation can be realized with a low calculation amount that makes complicated calculations unnecessary, using position curves mapped in the distance space recorded in advance in the memory. Because of low computational complexity, even inexpensive microcomputers can be dealt with, and even wearable terminals with low battery capacity can operate for a long time.

\section{AUTOMATIC GUIDANCE SYSTEM}

\subsection{System Configuration}

Fig 1 illustrates the system configuration of the smart mobile terminal guide system. As shown in Fig 1, there are two main area, one is facilities management department, another is exhibition facilities. In the facilities management department, content editor provides content creation and editing and coding, and tourist usage history analysis application aggregates the behavior of tourists, and integrated device management station is used for setting content codes to the device and charging the device and read out the history data from the device. In exhibition facilities, several PAN tags or
IR tags are located in exhibition area to indicate the location of the exhibit. The wearable devices are used to automatically play the guidance contents of the exhibition for tourists, when the wearable device approaches an exhibition. For the wearable is equipped on the visitor's head, the Mutual position between the device and user is fixed or constrained. Therefore, the orientation of the device can be used to show the orientation of the visitor.
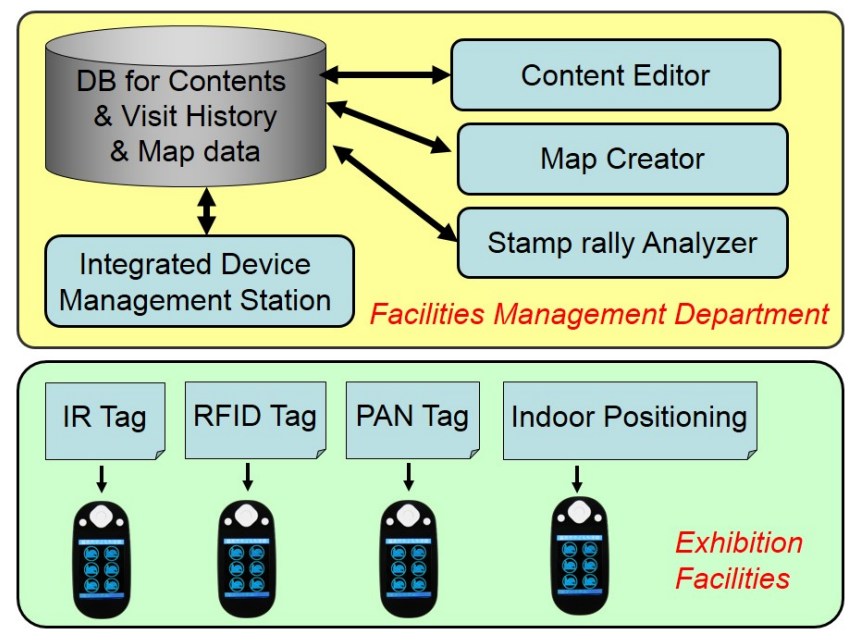

Fig. 1. Total Configuration of Guide System

\subsection{Principle of Positioning by Ultrasonic Wave}

Fig 2 shows the model of positioning using three ultrasonic transmitters. In the area of positioning, ultrasonic transmitters are installed at minimum three different places in order to obtain the coordinates of the receiving terminal.@The coordinates are shown as $(\mathrm{x}, \mathrm{y}, \mathrm{z})$. The coordinates of the transmitter $\mathrm{i}$, and the distance between the receiving node and the transmitter? $i$ are shown as $\left(x_{t_{i}}, y_{t_{i}}, z_{t_{i}}\right)$ and $r_{i}$ respectively. Here, $i=1,2,3$. Based on the triangular positioning principle, the position of the receiving node $(x, y, z)$ should be obtained from the following equations.

$$
\begin{aligned}
& \left(x-x_{t_{1}}\right)^{2}+\left(y-y_{t_{1}}\right)^{2}+\left(z-z_{t_{1}}\right)^{2}=r_{1}^{2} \\
& \left(x-x_{t_{2}}\right)^{2}+\left(y-y_{t_{2}}\right)^{2}+\left(z-z_{t_{2}}\right)^{2}=r_{2}^{2} \\
& \left(x-x_{t_{3}}\right)^{2}+\left(y-y_{t_{3}}\right)^{2}+\left(z-z_{t_{3}}\right)^{2}=r_{3}^{2}
\end{aligned}
$$

The distances $r_{1}, r_{2}, r_{3}$ can be measured from the propagation delay time of ultrasonic wave. Here the propagation delay time is $\delta t_{i}$ and the sound speed is $v$, then the distance $r_{i}$ can be calculated by $r_{i}=\delta t_{i} v$ simplely. To measure the propagation delay, we use a radio beacon to trigger the start of a ultrasonic emission, the receiver will begin to count the time of the ultrasonic arriving. Generally, if the above simultaneous equations are numerically solved by the Newton method or the like, the coordinates of the receiving node can be calculated.

However, in the case of using an inexpensive low-end mobile terminal, the poor real time property due to long computation time is a problem due to un-specificity from the selection of the initial value and a large amount of calculation. Speeding up by increasing the frequency of the SoC can shorten the calculation time, but the 


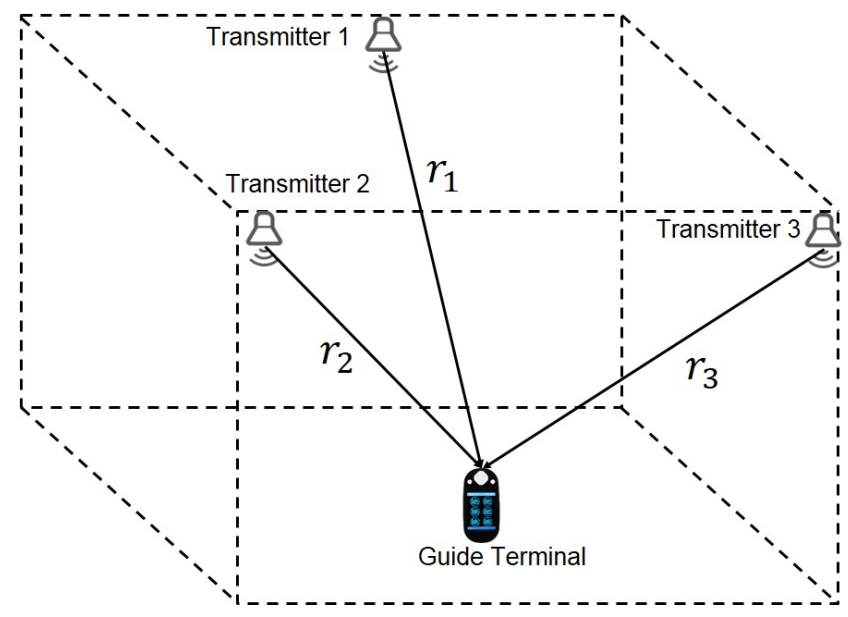

Fig. 2. Indoor positioning layout of the guide system

consumption of the battery is intense and the terminal work time will be greatly shortened.

\subsection{Distance Measurement System by Ultrasound}

In this research, each transmitter transmits ultrasonic waves of a certain frequency. The outline of the proposed system is shown in Fig 2 In order to measure the propagation time, it is necessary to synchronize the time with the transmitter and the receiver. Here, the measurement time is synchronized by using the wireless tag equipped with the PAN communication. Immediately before sending an ultrasonic wave from the transmitter, the wireless tag connected to the transmitter transmits a wireless synchronization signal to the receiver. When the receiver gets a synchronization signal from the transmitter, it will begin to detect the ultrasonic wave and measure the period time from the time of the synchronization signal. With this period time, we can calculate the distance from the transmitter by multiplying the speed of the ultrasonic wave. Once the distances from all at least three transmitters are calculated, the position of the receiver may be calculated using the above equations.

\subsection{Acceleration Approach of Position Calculation}

At present, as a general position calculation method, the position of the terminal is calculated using the numerical calculation algorithm of the above nonlinear simultaneous equation set. As a problem of this method, it is a computation amount by a complicated calculation process, a long calculation time and a large power consumption. It is difficult to use this in a mobile information terminal or wearable terminal.@

In the current method, the position of the receiving terminal is calculated using the distance between the receiving terminal and each transmitting terminal. The coordinates of the receiver node can be shown in the following function mapping.

$$
\begin{aligned}
& x=f_{x}\left(r_{1}, r_{2}, r_{3}\right) \\
& y=f_{y}\left(r_{1}, r_{2}, r_{3}\right) \\
& z=f_{z}\left(r_{1}, r_{2}, r_{3}\right)
\end{aligned}
$$

The mapping satisfies the above nonlinear equations. Computing time of the mapping is long from low end SoC. Therefore, it is difficult to implement the approach to mobile terminal application such as, exhibition guide service, which need a frequent position confirmation and good real time performance.

If the position of the guide terminal is known as $(x, y, z)$, then the distances to each transmitter can be calculated by

$$
\begin{aligned}
& r_{1}=\sqrt{\left(x-x_{t_{1}}\right)^{2}+\left(y-y_{t_{1}}\right)^{2}+\left(z-z_{t_{1}}\right)^{2}} \\
& r_{2}=\sqrt{\left(x-x_{t_{2}}\right)^{2}+\left(y-y_{t_{2}}\right)^{2}+\left(z-z_{t_{2}}\right)^{2}} \\
& r_{3}=\sqrt{\left(x-x_{t_{3}}\right)^{2}+\left(y-y_{t_{3}}\right)^{2}+\left(z-z_{t_{3}}\right)^{2}}
\end{aligned}
$$

If we can mapping the spot area to a distance space in advance, we may confirm the position using only the distance information $\left(r_{1}, r_{2}, r_{3}\right)$. With this approach, we do not need to solve such nonlinear equation online, so the calculation can be reduced greatly. Therefore, a simple calculation for position detection can be run at a very frequent to provide a good real time performance for visitors who are walking in exhibition area

To realize the above approach, it is import to save the spot area information that is easy to confirm. In exhibition guide application, it is often to set a geometric shape area. When a visitor who hold an exhibition guide enters the area, the exhibition guide will start an explanation about the exhibition. In general, the shape of the area is often defined as a round shape or rectangle for a simple computation by confirming the following conditions

$$
\left(x-x_{d}\right)^{2}+\left(y-y_{d}\right)<M^{2}
$$

Or

$$
\begin{array}{r}
\left|x-x_{d}\right|<X_{d} \\
\left|y-y_{d}\right|<Y_{d}
\end{array}
$$

If we use distances to detect the spot area, we need to transform the above conditions in distance space. For the mapping between distance space and exhibition space is nonlinear mapping, so it is not possible to have same style condition as above.

In order to efficiently detect the spot area with the distances information, we present a numerical model to represent the spot area in distance space.

The approach consists of two stages. The first stage is carried out offline to construct a spot numerical model DB in distance space. The second stage is to search a spot online with the distance information.

In offline DB construction process, each spot is assigned a unique ID to show its identification. For each spot, the spot area is divided into grids, then calculate the distance with formula (7), (8) and (9) for each grid of the spot area. The record in the DB is defined as following format: $\left(r_{1}^{i}, r_{2}^{i}, r_{3}^{i}\right)$, here, $i=1,2, \cdots, N, N$ is the total grid number. In order to realize an efficient search process, here we introduce two characteristic parameters: a geometric center of spot mapping in distance space, and spot area boundary norm.

This DB data includes spot ID, a geometric center of spot mapping in distance space, and spot area boundary norm. The geometric center $\phi=\left(\phi_{r_{1}}, \phi_{r_{2}}, \phi_{r_{3}}\right)$ is given as

$$
\begin{aligned}
\phi_{r_{1}} & =\frac{\sum_{i=1}^{N} r_{1}^{i}}{N} \\
\phi_{r_{2}} & =\frac{\sum_{i=1}^{N} r_{2}^{i}}{N}
\end{aligned}
$$




$$
\phi_{r_{3}}=\frac{\sum_{i=1}^{N} r_{3}^{i}}{N}
$$

Here, $N$ is the total number of the grids in the spot area with $I D$. The spot area boundary norm $\Gamma$ is defined as

$$
\Gamma=\max _{1 \leq i \leq N}\left\{\sqrt{\left(r_{1}^{i}-\phi_{r_{1}}\right)^{2}+\left(r_{2}^{i}-\phi_{r_{2}}\right)^{2}+\left(r_{3}^{i}-\phi_{r_{3}}\right)^{2}}\right\}
$$

The flow chart of the first stage is shown in Fig 3 The processing calculation of this stage will be carried out offline. After the construction of the $\mathrm{DB}$, the $\mathrm{DB}$ data will be transmitted to the terminal for online use.

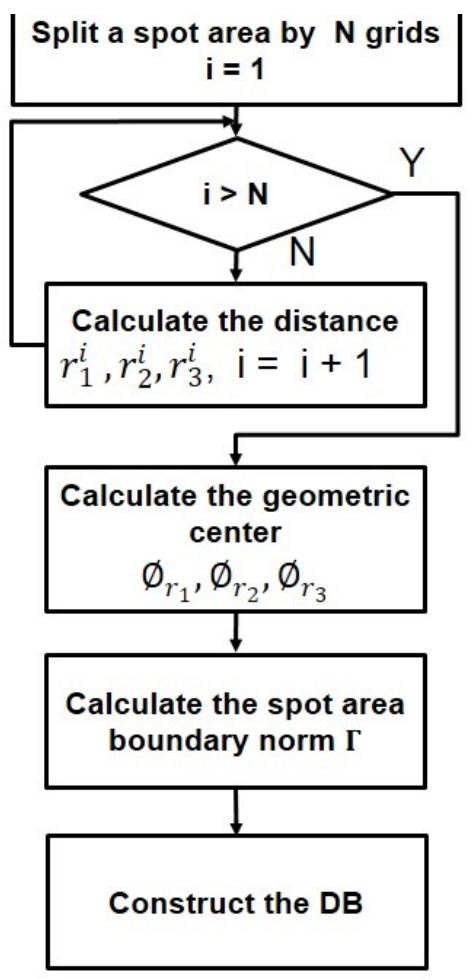

Fig. 3. Approach for building the numerical DB

In second stage, the distance information measured from the receiver will be used to detect if the terminal is in spot area that is defined in the numerical DB. In this process, the following calculation will be done with a measured vector $\left(r_{1}, r_{2}, r_{3}\right)$ at first step.

$$
\xi=\sqrt{\left(r_{1}-\phi_{r_{1}}\right)^{2}+\left(r_{2}-\phi_{r_{2}}\right)^{2}+\left(r_{3}-\phi_{r_{3}}\right)^{2}}
$$

At second step, the following condition will be checked to filter the points that may not be possilbe as a point in spot area.

$$
\xi<\Gamma
$$

At third step, we introduce a minimum distance norm to check if the measured distance vector represents a point in the spot area. The minimum distance norm is defined as

$$
\Psi=\min _{1 \leq i \leq N}\left\{\sqrt{\left(r_{1}^{i}-r_{1}\right)^{2}+\left(r_{2}^{i}-r_{2}\right)^{2}+\left(r_{3}^{i}-r_{3}\right)^{2}}\right\}
$$

Then the following condition will be checked to identify if the point is in the spot area.

$$
\Psi<\epsilon
$$

The point will be identified as a point in the spot area, If condition (20) is satisfied. $\epsilon$ is a very small positive number that can be decided in the first stage while building the numerical DB. The process of the second stage that is carried out online is shown as in Fig 4

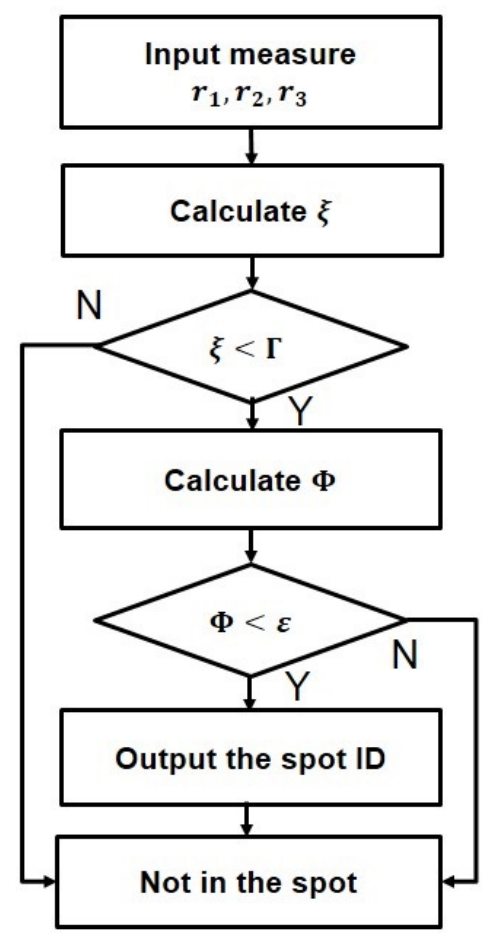

Fig. 4. Approach to detect the spot area in distance space

\section{SIMULATION EXPERIMENT}

In order to confirm the performance of the distance space approach proposed in this research, several numerical simulation was carried out at Matlab environment. The evaluation environment for positioning simulation is shown in Fig 5 As shown in Fig 5 , the exhibition space is $2000 \mathrm{~cm}$ in width and $3000 \mathrm{~cm}$ in length and $300 \mathrm{~cm}$ in height. Three ultrasonic transmitters are set in three positions, and the coordinates of the transmitters are $T_{1}=$ $(1500,1500,300), T_{2}=(0,0,300), T_{3}=(3000,0,300)$. In this exhibition space, a square spot areas $S_{1}$ is set for guide service. The geometric coordinates of these spot area shown in Fig 5 is (2000, 1000), (2100, 1000), (2100, 1100), (2000, 1100). The spot area $S_{1}$ is split by $10 \times 10$ grids. Using the approach shown in Fig 3 the DB for the spot area $S_{1}$ can be obtained. The geometric center of spot mapping is $\phi=(1108.4,2308.4,1424.3)$, and the spot area boundary norm is $\Gamma=100.13$. There are 100 mapping vectors in the $\mathrm{DB}$ for the spot. To save the $\mathrm{DB}$ data, less than $0.5 \mathrm{kB}$ is necessary if using $32 \mathrm{bit}$ float. 


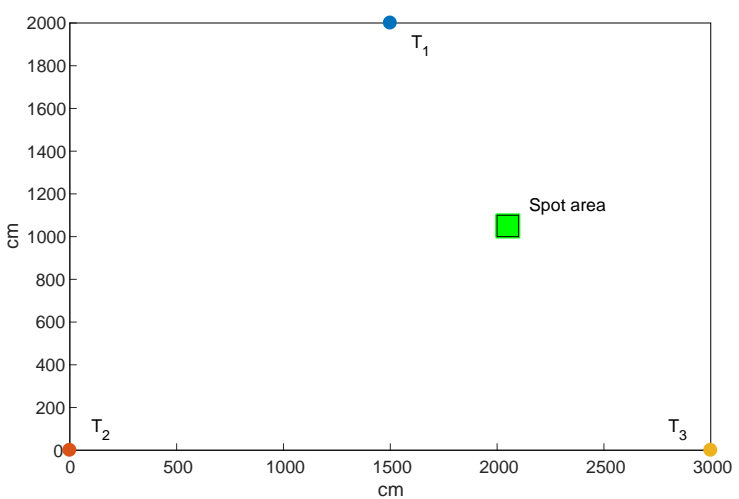

Fig. 5. layout of the exhibition environment for simulation

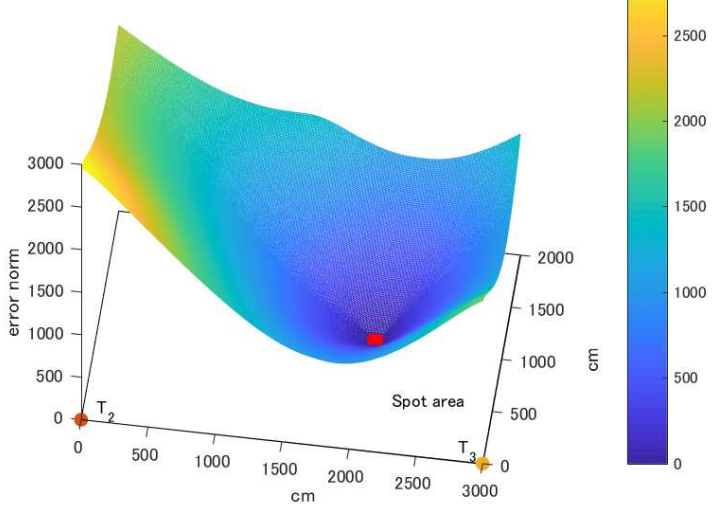

Fig. 6. Simulation result of error index distribution

Fig 6 shows the error index distribution for all exhibition area. As shown in Fig 6, the error index calculated from (??) increases rapidly, while the point is away from the $S_{1}$. Therefore, the error index present here is a useful index for detecting the spot area.

With the appraoch shown in Fig 4 we can check if a point in the exhibition space is in the spot area $S_{1}$.

At first, condition (18) will be checked for selecting candidate ones. The cyan color area in Fig 7 shows the candidate points that may be in spot area $S_{1}$.

For these points, further detecting process will be carried out by use of (19). For the points that are outside the cyan area in Fig 7 . no further process is necessary. This will reduce the computation greatly compared with existing appraoches. For points inside the cyan area, computation by $(19)$ is necessary, but they are simple computation. If the data in the DB is sort at a special order, the performance of searching process for condition $[20$ will be improved greatly.

The red color area in Fig 8 shows the points that are recognized as points in the spot area $S_{1}$. All the points in spot area are identified correctly, but some points just outsise spot area boundary are identified as spot area points incorrently. For this case, the

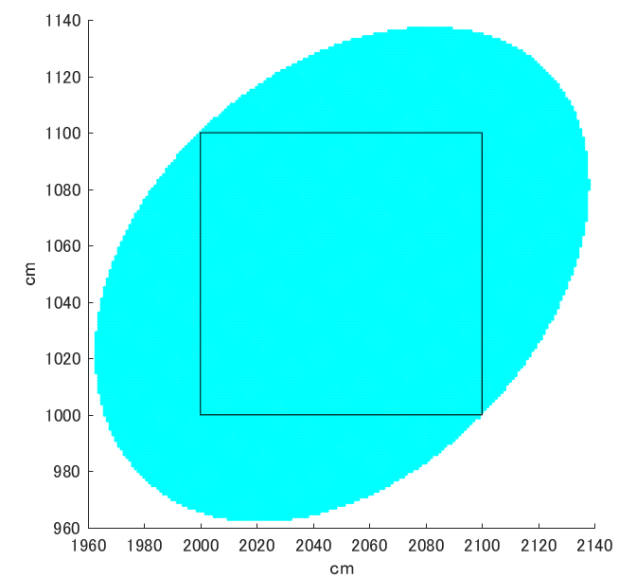

Fig. 7. Points to be a candidate for the spot area

error distance is less than $5 \mathrm{~cm}$. Such error level can be ignored for the measure error of ultrasonic wave is about $30 \mathrm{~cm}$ in general. Also, this error may be reduced by modification calculation at the numerical DB building.

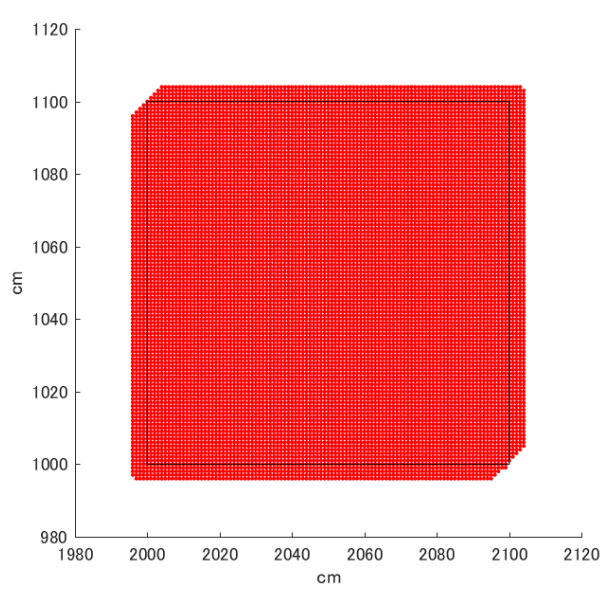

Fig. 8. Points recognized in the spot area

\section{CONCLUSIONS}

TOA is known as a most popular approach for positioning system, but it is necessary to solve nonlinear simultaneous equation that is a very time consumption and complexity process. Therefore, it is difficult to implement the approach to smart device that may have cheap SoC or smaller battery. In order to provide indoor positioning for real-time guide service that is on a moible terminal platform, a new indoor positioning approach is proposed in this paper.

With use of ultrasonic wave. Ultrasonic approach may bring a better measure precision distance than other approaches, so ultrasonic measurement is used in our exhibition guide system to identify the position of the guide device. To avoid the complex computation for solving the nonlinear equation, we introduced a new concept of distance space and proposed a offline and online 
hybrid computation architecture to reduce the computation time greatly. Offline processing will build a numerical DB for spot area that may be complex computation, and online computation is only for detecting the spot area with very simple computation. Offline computation can be carried out in high performance personal computer, and send the result to mobile terminal for online use. The online computation is run in an embedded platform that may have less computation ability.

The result of simulation experiment with the new distance space approach demonstrates a good performance both in efficient and accuracy. The approach presnet in the research may be expected to have a wide use for mobile service.

\section{REFERENCES}

[1] C. Huang, H. Manh, RSS-based indoor positioning based on multi-dimensional kernel modeling and weighted average tracking,IEEE Sensors Journal, Vol.16, No.9, pp.3231-3245, 2016

[2] S. Murata, C. Yara, K. Kaneta, S. Ioroi, H. Tanaka, Accurate indoor positioning system using near-ultrasonic sound from a smartphone, Proceedings of the 8th International Conference on Next Generation Mobile Applications, Services and Technologies (NGMAST '14), pp.13-18, September 2014

[3] A. De Angelis, A. Moschitta, P. Carbone et al., Design and characterization of a portable ultrasonic indoor 3-d positioning system, IEEE Transactions on Instrumentation and Measurement, Vol.64, No.10, pp.2616-2625, 2015

[4] Dawei Cai, Yuji Saito and Yoshihiko Abe, An Information Broadcasting System with Infrared data Communication Protocol, WSEAS Trans. on Communication, Vol.2(3), 228234, 2003

[5] P. Cotera, M. Velazquez, D. Cruz, L. Medina, M. Bandala, Indoor robot positioning using an enhanced trilateration algorithm, International Journal of Advanced Robotic Systems, Vol. 13, No.3, p.110, 2016

[6] Dawei Cai, Realization of Autonomous Guidance Service by Integrating Information from NFC and MEMS, Proceedings of International Conference on Software Design Engineering, 2014

[7] E. Laitinen, E. S. Lohan, On the choice of access point selection criterion and other position estimation characteristics for WLAN-based indoor positioning, Sensors, Vol.16, No.5, p.737, 2016

[8] J. Rapinski and S. Cellmer, Analysis of range based indoor positioning techniques for personal communication networks, Mobile Networks and Applications, Vol. 21, No. 3, p.539549, 2016

[9] P. Cotera, M. Velazquez, D. Cruz, L. Medina, and M. Bandala, Indoor robot positioning using an enhanced trilateration algorithm, International Journal of Advanced Robotic Systems, Vol.13, No.3, p.110, 2016

[10] T. Haute, E. Poorter, P. Crombez et al., Performance analysis of multiple Indoor Positioning Systems in a healthcare environment, International Journal of Health Geographics, Vol.15, article 7, 2016

[11] A. De Angelis, A. Moschitta, P. Carbone et al., Design and characterization of a portable ultrasonic indoor 3-d positioning system, IEEE Transactions on Instrumentation and Measurement, Vol.64, No.10, p.2616-2625, 2015 